\title{
Randall-Sundrum model with a small curvature and dielectron production at the $\mathrm{LHC}$
}

\author{
A.V. Kisselev* \\ Institute for High Energy Physics, 142281 Protvino, Russia \\ and \\ Department of Physics, Moscow State University, 119991 Moscow, Russia
}

\begin{abstract}
In the framework Randall-Sundrum-like scenario with the small curvature $\kappa$ (RSSC model), $p_{\perp}$-distributions for the dielectron production at the LHC are calculated. For the summary statistics taken at $7 \mathrm{TeV}\left(L=5 \mathrm{fb}^{-1}\right)$ and $8 \mathrm{TeV}\left(L=20 \mathrm{fb}^{-1}\right)$, the exclusion limit on the 5-dimensional gravity scale $M_{5}$ is found to be $6.35 \mathrm{TeV}$ at $95 \%$ C.L. For $\sqrt{s}=13 \mathrm{TeV}$ and integrated luminosity $30 \mathrm{fb}^{-1}$, the LHC search limit is estimated to be $8.95 \mathrm{TeV}$. These limits on $M_{5}$ are independent of $\kappa$, provided the relation $\kappa \ll M_{5}$ is satisfied.
\end{abstract}

\section{Dielectron production in the RSSC model}

In a recent paper [1, the $p_{\perp}$-distributions for the dimuon production at the LHC were calculated in the framework of the Randall-Sundrum-like scenario with the small curvature (RSSC model [2]-4]). The LHC discovery limits on 5-dimensional gravity scale $M_{5}$ were obtained for both $7 \mathrm{TeV}$ and $14 \mathrm{TeV}$.

In contrast to the RS1 model [5], in the RSSC model the masses of the Kaluza-Klein $(\mathrm{KK})$ excitations $h_{\mu \nu}^{(n)}(x)$ are proportional to the curvature parameter $\kappa\left(\kappa \ll M_{5}\right)$ [3],

$$
m_{n}=x_{n} \kappa, \quad n=1,2, \ldots,
$$

*Electronic address: alexandre.kisselev@ihep.ru 
where $x_{n}$ are zeros of the Bessel function $J_{1}(x)$. The interaction of the gravitons with the SM fields is described by the Lagrangian

$$
\mathcal{L}_{\mathrm{int}}=-\frac{1}{\bar{M}_{\mathrm{Pl}}} h_{\mu \nu}^{(0)}(x) T_{\alpha \beta}(x) \eta^{\mu \alpha} \eta^{\nu \beta}-\frac{1}{\Lambda_{\pi}} \sum_{n=1}^{\infty} h_{\mu \nu}^{(n)}(x) T_{\alpha \beta}(x) \eta^{\mu \alpha} \eta^{\nu \beta}
$$

where $T_{\mu \nu}(x)$ is the energy-momentum tensor of the SM matter,

$$
\Lambda_{\pi} \simeq \bar{M}_{\mathrm{Pl}} e^{-\pi \kappa r_{c}}
$$

and $\bar{M}_{\mathrm{Pl}}$ is the reduced Planck mass.

The goal of this paper is to estimate gravity effects in the dielectron production,

$$
p p \rightarrow e^{+} e^{-}+X,
$$

at LHC energies in the RSSC model. The differential cross section of the process (41) is given by

$$
\begin{aligned}
\frac{d \sigma}{d p_{\perp}}\left(p p \rightarrow e^{+} e^{-}+X\right) & =2 p_{\perp} \sum_{a, b=q, \bar{q}, g} \int \frac{d \tau \sqrt{\tau}}{\sqrt{\tau-x_{\perp}^{2}}} \int \frac{d x_{1}}{x_{1}} f_{a / p}\left(\mu^{2}, x_{1}\right) \\
& \times f_{b / p}\left(\mu^{2}, \tau / x_{1}\right) \frac{d \sigma}{d \hat{t}}\left(a b \rightarrow e^{+} e^{-}\right)
\end{aligned}
$$

with the transverse energy of the electron pair equals to $2 p_{\perp}$. In eq. (15) two dimensionless quantities are introduced

$$
x_{\perp}=\frac{2 p_{\perp}}{\sqrt{s}}, \quad \tau=x_{1} x_{2}
$$

where $x_{2}$ is the momentum fraction of the parton $b$ in (5). Without cuts, integration variables in (5) vary within the following limits

$$
x_{\perp}^{2} \leq \tau \leq 1, \quad \tau \leq x_{1} \leq 1 .
$$

After imposing kinematical cut on electron rapidity, the integration region becomes more complicated (see Appendix A in ref. [1]).

The SM contribution to the $p_{\perp}$-distribution looks like

$$
\frac{d \sigma^{\mathrm{SM}}}{d \hat{t}}\left(q \bar{q} \rightarrow e^{+} e^{-}\right)=\frac{1}{48 \pi s^{2}}\left[u^{2}\left(\left|G^{L L}\right|^{2}+\left|G^{R R}\right|^{2}\right)+t^{2}\left(\left|G^{L R}\right|^{2}+\left|G^{R L}\right|^{2}\right)\right]
$$


with

$$
G^{A B}(s)=\sum_{V=\gamma, Z} \frac{g_{A}\left(V \rightarrow e^{+} e^{-}\right) g_{A}(V \rightarrow q \bar{q})}{s-m_{V}^{2}+i m_{V} \Gamma_{V}} .
$$

Here $g_{L(R)}\left(\gamma \rightarrow e^{+} e^{-}\right)=g_{L(R)}(\gamma \rightarrow q \bar{q})=e$, and

$$
\begin{aligned}
g_{L}\left(Z \rightarrow e^{+} e^{-}\right) & =-\frac{1}{2}+\sin ^{2} \theta_{W}, \\
g_{R}\left(Z \rightarrow e^{+} e^{-}\right) & =\sin ^{2} \theta_{W}, \\
g_{L}(Z \rightarrow q \bar{q}) & =T_{3}^{q}-e_{q} \sin ^{2} \theta_{W}, \\
g_{R}(Z \rightarrow q \bar{q}) & =-e_{q} \sin ^{2} \theta_{W},
\end{aligned}
$$

with $T_{3}^{q}$ being third component of the quark isospin, $e_{q}$ being quark electric charge (in units of $|e|$ ).

The graviton contribution comes from both quark-antiquark annihilation and gluon-gluon fusion subprocesses (see, for instance, [2])

$$
\begin{aligned}
\frac{d \sigma^{\text {grav }}}{d \hat{t}}\left(q \bar{q} \rightarrow e^{+} e^{-}\right) & =\frac{\hat{s}^{4}+10 \hat{s}^{3} \hat{t}+42 \hat{s}^{2} \hat{t}^{2}+64 \hat{s} \hat{t}^{3}+32 \hat{t}^{4}}{1536 \pi \hat{s}^{2}}|\mathcal{S}(\hat{s})|^{2}, \\
\frac{d \sigma^{\text {grav }}}{d \hat{t}}\left(g g \rightarrow e^{+} e^{-}\right) & =-\frac{\hat{t}(\hat{s}+\hat{t})\left(\hat{s}^{2}+2 \hat{s} \hat{t}+2 \hat{t}^{2}\right)}{256 \pi \hat{s}^{2}}|\mathcal{S}(\hat{s})|^{2}
\end{aligned}
$$

where

$$
\mathcal{S}(s)=\frac{1}{\Lambda_{\pi}^{2}} \sum_{n=1}^{\infty} \frac{1}{s-m_{n}^{2}+i m_{n} \Gamma_{n}}
$$

is the invariant part of the partonic matrix elements, with $\Gamma_{n}$ being total width of the graviton with the KK number $n$ and mass $m_{n}$ [4]:

$$
\Gamma_{n}=\eta m_{n}\left(\frac{m_{n}}{\Lambda_{\pi}}\right)^{2}, \quad \eta \simeq 0.09 .
$$

Let us note that $\mathcal{S}(s)$ is a universal function for processes mediated by $s$ channel virtual gravitons.

In the RSSC model, an explicit expression for the sum (10) was obtained in [4],

$$
\mathcal{S}(s)=-\frac{1}{4 \bar{M}_{5}^{3} \sqrt{s}} \frac{\sin 2 A+i \sinh 2 \varepsilon}{\cos ^{2} A+\sinh ^{2} \varepsilon},
$$


where $\bar{M}_{5}=M_{5} /(2 \pi)^{1 / 3}$ is the reduced 5-dimensional gravity scale, and

$$
A=\frac{\sqrt{s}}{\kappa}, \quad \varepsilon=\frac{\eta}{2}\left(\frac{\sqrt{s}}{\bar{M}_{5}}\right)^{3} .
$$

Let us underline that the magnitude of $\mathcal{S}(s)$ is defined by the scale $\bar{M}_{5}$, not by the coupling $\Lambda_{\pi}$ in the Lagrangian (2). In general, this property is valid in the RSSC model for both real and virtual production of the KK gravitons [3, 4].

\section{Numerical calculation of $\mathbf{p}_{\perp}$ distributions}

Taking into account that the transition region $1.44<|\eta|<1.57(1.37<|\eta|<$ 1.52) between the ECAL barrel and endcap calorimeters is usually excluded in the CMS (ATLAS) experiment, we impose the CMS cut on the electron pseudorapidity,

$$
|\eta|<1.44, \quad 1.57<|\eta|<2.50 .
$$

The reconstruction efficiency $85 \%$ is assumed for the dielectron events [6]. We use the MSTW NNLO parton distributions [7], and convolute them with the partonic cross sections. The PDF scale is taken to be equal to the invariant mass of the electron pair, $\mu=M_{e^{+} e^{-}}$. In order to take into account SM higher order corrections, the $K$-factor 1.5 is used for the SM background, while a conservative value of $K=1$ is taken for the signal.

The differential cross section of the process under consideration has three terms

$$
d \sigma=d \sigma(\mathrm{SM})+d \sigma(\text { grav })+d \sigma(\mathrm{SM}-\operatorname{grav})
$$

where the last one comes from the interference between the SM and graviton interactions. Since the SM amplitude is pure real, while the real part of each graviton resonance is antisymmetric with respect to its central point, the interference term has appeared to be negligible in comparison with the pure gravity and SM terms after integration in partonic momenta.

The account of the graviton widths is a crucial point for both analytical calculations and numerical estimations. As it was shown in refs. [1], [8, an ignorance of the graviton widths is a rough approximation, since it results in very large suppression of the cross sections. The reason lies partially in the fact that

$$
\frac{d \sigma(\text { grav })}{d p_{\perp}} \sim \frac{1}{p_{\perp}^{3}}\left(\frac{\sqrt{s}}{\bar{M}_{5}}\right)^{3}
$$


while in zero width approximation one gets

$$
\left.\frac{d \sigma(\text { grav })}{d p_{\perp}}\right|_{\text {zero width }} \sim \frac{1}{\bar{M}_{5}^{3}}\left(\frac{\sqrt{s}}{\bar{M}_{5}}\right)^{3} .
$$

Let us stress that in the RSSC model the gravity cross sections do not depend on the curvature $\kappa$ (up to small power corrections), provided $\kappa \ll \bar{M}_{5}$, in contrast to the RS1 model [5] in which all bounds on $\bar{M}_{5}$ depend on the ratio $\kappa / \bar{M}_{\mathrm{Pl}}$.

In Fig. 1 the results of our calculations of gravity cross sections for the dielectron production at $8 \mathrm{TeV} \mathrm{LHC}$ are presented. The differential cross

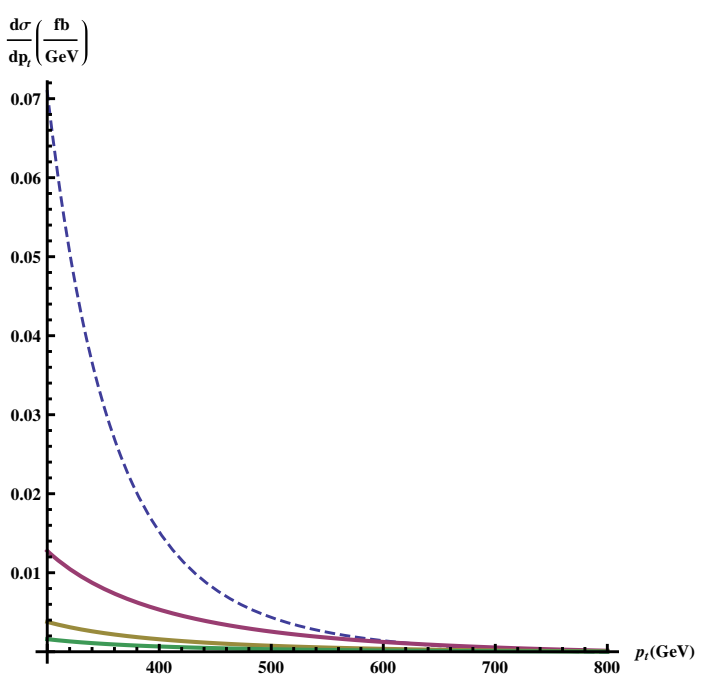

Figure 1: The KK graviton contribution to the dielectron production for $\bar{M}_{5}=2,4,6 \mathrm{TeV}$ (solid curves, from above) vs. SM (Born) contribution (dashed curve) at $\sqrt{s}=8 \mathrm{TeV}$.

sections for $13 \mathrm{TeV}$ are shown in Fig. 2. Note that the gravity mediated contributions to the cross sections do not include the SM contribution (i.e. solid lines in the figures correspond to pure gravity contributions).

Let $N_{S}\left(N_{B}\right)$ be a number of signal (background) dielectron events with $p_{\perp}>p_{\perp}^{\text {cut }}$,

$$
N_{S}=\int_{p_{\perp}>p_{\perp}^{\text {cut }}} \frac{d \sigma(\text { grav })}{d p_{\perp}} d p_{\perp}, \quad N_{B}=\int_{p_{\perp}>p_{\perp}^{\text {cut }}} \frac{d \sigma(\mathrm{SM})}{d p_{\perp}} d p_{\perp} .
$$




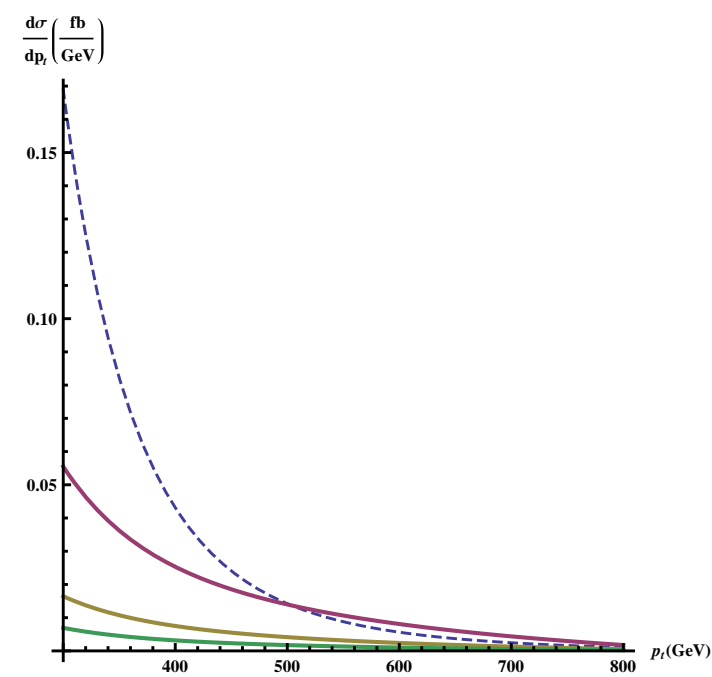

Figure 2: The KK graviton contribution to the dielectron production for $\bar{M}_{5}=4,6,8 \mathrm{TeV}$ (solid curves, from above) vs. SM (Born) contribution (dashed curve) at $\sqrt{s}=13 \mathrm{TeV}$.

Then we define the statistical significance $\mathcal{S}=N_{S} / \sqrt{N_{B}+N_{S}}$, and require a $5 \sigma$ effect. In figure 3 the statistical significance is shown for total number of "events" with $\sqrt{s}=7 \mathrm{TeV}$ and $\sqrt{s}=8 \mathrm{TeV}$ as a function of the transverse momentum cut $p_{\perp}^{\text {cut }}$ and reduced 5 -dimensional gravity scale $\bar{M}_{5}$. The integrated luminosity was taken to be $5 \mathrm{fb}^{-1}$ and $20 \mathrm{fb}^{-1}$ for $\sqrt{s}=7$ $\mathrm{TeV}$ and $\sqrt{s}=8 \mathrm{TeV}$, respectively. Figure 4 represents the significance $\mathcal{S}$ for the dielectron events with $\sqrt{s}=13 \mathrm{TeV}$ and $30 \mathrm{fb}^{-1}$.

Previously, calculations of dilepton cross sections were done in [2] without taking into account finite widths of the KK gravitons. As was shown in [1] (see also [8]), in zero width approximation the gravity cross sections are very small in comparison with the background cross section at low and moderate values of $p_{\perp}$. That is why, a high cut $p_{\perp}^{\text {cut }}$ is needed in order to get $N_{S}$ comparable with $N_{B}$. Correspondingly, LHC search limits in [2] are significantly smaller than in non-zero approximation for the graviton widths. 


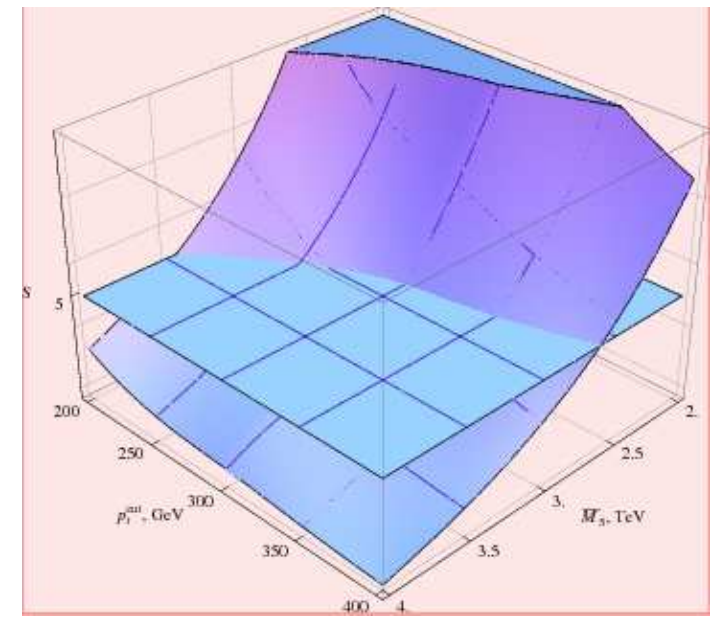

Figure 3: The statistical significance $S$ for the dielectron production at the LHC for $\sqrt{s}=(7+8) \mathrm{TeV}$ and integrated luminosity $(5+20) \mathrm{fb}^{-1}$ as a function of the transverse momentum cut $p_{\perp}^{\text {cut }}$ and reduced 5-dimensional gravity scale $\bar{M}_{5}$. The plane $\mathcal{S}=5$ is also shown.

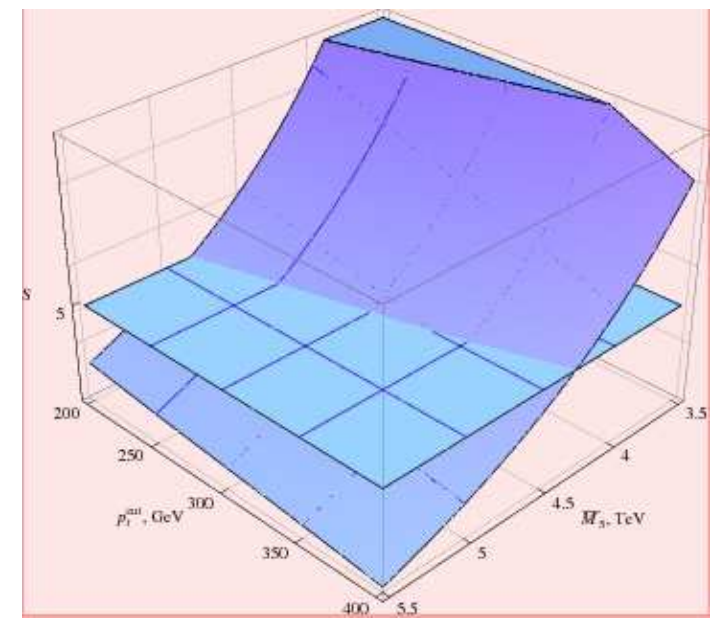

Figure 4: The same as in figure 3, but for $\sqrt{s}=13 \mathrm{TeV}$ and integrated luminosity $30 \mathrm{fb}^{-1}$. 


\section{Conclusions}

In the present paper the RSSC model [3], [4] is considered in which the reduced 5-dimensional Planck scale $\bar{M}_{5}$ is much larger that the curvature $\kappa$. In such a model the mass spectrum and experimental signaturesare similar to those in the ADD model [9] with one flat extra dimension.

The $p_{\perp}$-distributions for the electron pairs production with high $p_{\perp}$ at the LHC are calculated for the collision energies $7 \mathrm{TeV}, 8 \mathrm{TeV}$ and $13 \mathrm{TeV}$, see Fig. 1 and Fig. 2 (figures for $7 \mathrm{TeV}$ are not shown). Let us underline that the account of the KK graviton widths was the crucial point for our calculations.

The statistical significance as a function of the reduced 5-dimensional Planck scale $\bar{M}_{5}$ and cut on the lepton transverse momentum $p_{\perp}^{\text {cut }}$ is calculated (Fig. 3 and Fig. 4). No significant deviations from the SM prediction were seen in the dielectron events at $(7+8) \mathrm{TeV}$ LHC with the integrated lu-

minosity $(5+20) \mathrm{fb}^{-1}$. By using our calculations, we come to the conclusion that the region

$$
M_{5}<6.35 \mathrm{TeV}
$$

is excluded by experimental data. We also obtain the discovery limit for the $13 \mathrm{TeV}$ LHC with the integrated luminosity $30 \mathrm{fb}^{-1}$,

$$
M_{5}=8.95 \mathrm{TeV} .
$$

Let us stress that these bounds on $M_{5}$ do not depend on the curvature $\kappa$ (up to small power-like corrections), contrary to the RS1 model [5] in which estimated bounds on $M_{5}$ significantly depend on the ratio $\kappa / \bar{M}_{\mathrm{Pl}}$.

\section{References}

[1] A.V. Kisselev, Randall-Sundrum model with a small curvature and dimuon production at the LHC, JHEP 04 (2013) 025 [arXiv:1210.3238].

[2] G. F. Giudice, T. Plehn and A. Strumia, Graviton collider effects in one and more large extra dimensions, Nucl. Phys. B 706 (2005) 455 hep-ph/0408320].

[3] A.V. Kisselev and V.A. Petrov, Gravireggeons and transplanckian scattering in models with one extra dimension, Phys. Rev. D 71 (2005) 124032 hep-ph/0504203. 
[4] A.V. Kisselev, Virtual gravitons and brane field scattering in the RS model with a small curvature, Phys. Rev. D 73 (2006) 024007 [hep-th/0507145].

[5] L. Randall and R. Sundrum, A large mass hierarchy from a small extra dimension, Phys. Rev. Lett. 83 (1999) 3370 [hep-ph/9905221].

[6] CMS collaboration, Search for large extra dimensions in dimuon and dielectron events in pp collisions at $\sqrt{s}=7$ TeV, Phys. Lett. B 711 (2012) 15 arXiv:1202.3827.

[7] A. Martin, W. Stirling, R. Thorne and G. Watt, Parton distributions for the LHC, Eur. Phys. J. C 63 (2009) 189 [arXiv:0901.0002].

[8] A.V. Kisselev, RS model with a small curvature and two-photon production at the LHC, JHEP 09 (2008) 039 arXiv:0804.3941.

[9] N. Arkani-Hamed, S. Dimopoulos and G. Dvali, The hierarchy problem and new dimensions at a millimeter, Phys. Lett. B 429 (1998) 263 hep-ph/9803315]; I. Antoniadis, N. Arkani-Hamed, S. Dimopoulos and G. Dvali, New dimensions at a millimeter to a Fermi and superstrings at a TeV, Phys. Lett. B 436 (1998) 257 hep-ph/9804398; N. ArkaniHamed, S. Dimopoulos and G. Dvali, Phenomenology, astrophysics and cosmology of theories with submillimeter dimensions and TeV scale quantum gravity, Phys. Rev. D 59 (1999) 086004 [hep-ph/9807344]. 